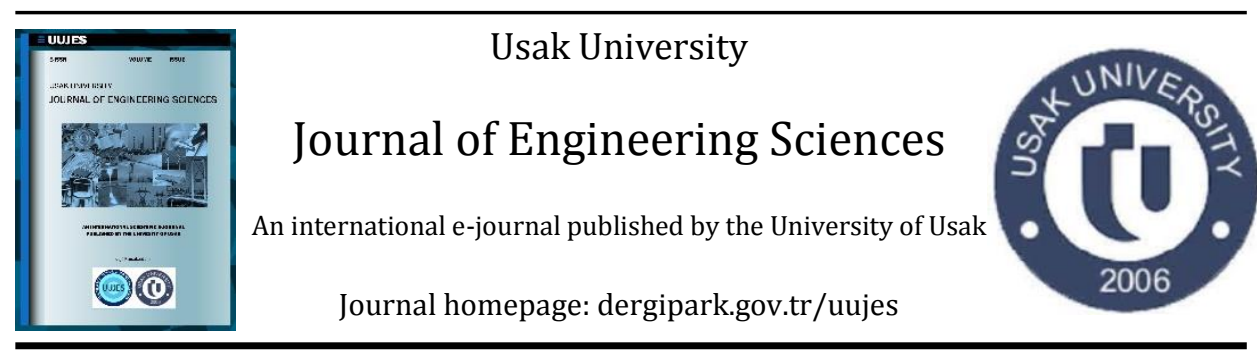

Research article

\title{
DESIGN AND SIMULATION ANALYSIS OF A TEXTILE BASED METAMATERIAL PERFECT ABSORBER FOR ENERGY HARVESTING APPLICATIONS AT WIFI BAND
}

\author{
Murat Ercan ${ }^{1}$, Gamze D. Tetik ${ }^{2 *}$, Muharrem Karaaslan 3 \\ ${ }^{1}$ Department of Materials Science and Nanotechnology Engineering, Graduate Education Institute, Usak \\ University, Turkey \\ ${ }^{2}$ Department of Materials Science and Nanotechnology Engineering Faculty of Engineering, Usak \\ University, Turkey \\ ${ }^{3}$ Department of Electrical and Electronics Engineering, Faculty of Engineering and Natural Sciences, \\ Iskenderun Technical University, Turkey
}

Received:17 December 2021 Revised:28 December 2021 Accepted:29 December 2021 Online available 30 December 2021 Handling Co-Editor: Fulya Yllmaz

\begin{abstract}
Metamaterials, which are called as left-handed materials, exhibit electromagnetic properties that do not exist in nature, such as negative refractive index and reverse Doppler effect, at the frequency range for which they are designed. These unique properties of metamaterials have made them indispensable in a wide variety of applications such as sensor applications, signal absorption, antenna, and energy harvesting. In this study, the design and analysis of a metamaterial (MTM) perfect absorber at $5 \mathrm{GHz}$ frequency band were performed to investigate the usability of the proposed structure in energy harvesting applications. A negative refractive index of -0.15 was obtained at $5 \mathrm{GHz}$. The variation of absorption, transmission, and reflection of the proposed MTM structure exhibited that a maximum absorbance was monitored at $5 \mathrm{GHz}$ as 0.9949 . According to the harvesting application study, the proposed structure has a maximum absorption of $88.3 \%$ at $4.98 \mathrm{GHz}$ which is close to resonant frequency in $8000 \Omega$ resistor value. The results of the study revealed that the proposed MTM structure has potentials to be used in energy harvesting applications.
\end{abstract}

Keywords: Metamaterials, energy harvesting, perfect absorber, wifi band, electronic textiles

(C)2021 Usak University all rights reserved.

*Corresponding author: Gamze D. Tetik

E-mail: gamze.tetik@usak.edu.tr (ORCID: 0000-0002-5968-7244)

DOI: $10.47137 /$ uujes.1037884

(C)2021 Usak University all rights reserved. 


\section{Introduction}

The rapid increase in the world population and the decrease in the energy resources direct researchers to work on energy. Recycling, renewable energy sources, advanced materials for energy applications, and energy harvesting are some hot topics of this area. Energy harvesting is known as the process of converting kinetic energies such as distortion energy, vibration, etc. into electrical energy [1]. The most used energy sources of the portable devices are batteries or battery-like devices. However, some disadvantages of these devices can be regarded as heaviness, big size, and rapid discharge. These disadvantages also prompted researchers to study on energy harvesting methods. Electromagnetic energy harvesting concerns with electromagnetic induction which is the electric potential generated in the conductor by the change of the magnetic field around it. Metamaterials (MTMs) are unique materials that can be utilized in electromagnetic energy harvesting applications. Their interesting features such as negative refractive index, negative dielectric constant, and negative magnetic permeability make them advantageous in various areas [2]. They are called as lefthanded materials and they are not found in nature per se.

The metamaterial foundations that were laid by Veselago about fifty years ago continued with experimental studies in the 2000s, and since then these unique materials have attracted academic interest $[3,4]$. The efficient preparation of these MTMs, in other words artificial electromagnetic structures, determines the EM (electromagnetic) properties [5] which are basically permittivity $\varepsilon(\omega)$ and permeability $\mu(\omega)$. These properties were determined by the structural design of the MTMs according to the applications. Till date plus [6], rectenna [7], C shaped rectangular [8], Hilbert [9], U and L [10], ring circle [11], serpentine [12], hegzagonal [13], octagonal [14] shaped MTM designs have been proposed for energy harvesting applications. Besides, conductive textile materials such as felt, zelt, and pure copper polyester taffeta fabrics (PCPTF) have been used as components of MTMs in various researches [15-17].

Several simulation and experimental studies have been conducted on MTMs for the EM harvesting in various operation frequency bands [18-21]. EM harvesting in radio frequency (RF) band was conducted by Kaur et al. with an ultrathin dual layer MTM design. They observed $76 \%$ and $94 \%$ absorption values for incidence angle up to $45^{\circ}$ in transverse electric and $60^{\circ}$ in transverse magnetic operation modes, respectively [22]. Coskuner and Garcia-Garcia proposed metamaterial-based transmission lines to improve the operation frequencies to 2.4- $5 \mathrm{GHz}$ [23]. Another group conducted a simulation study about microwave energy harvesting and their results showed that the power absorption efficiency of the dual polarized single cell was $98 \%$ at $2 \mathrm{GHz}$ [24]. Moreover, the harvesting efficiency of $96.5 \%$ at $2.45 \mathrm{GHz}$ was calculated via the simulation results of a $9 \times 9$ fractal based MTM structure. The authors emphasized the potentials of the proposed harvesters for wireless sensor network applications [25]. Umaña-Idarraga et al. proposed a multi resonant MTM structure having absorption efficiencies of $98.2 \%$ and $99.7 \%$ at 2.4 and $4.2 \mathrm{GHz}$, respectively [26]. Another group working on the GSM and WIFI bands stated that the harvesting efficiency of their MTM structure was around $90 \%$ for the 2.60 and $5.80 \mathrm{GHz}$ frequencies when the incidence angle was $30^{\circ}[2]$.

The wireless local area network (WLAN) operations cover the industrial, scientific, and medical (ISM) frequency band (2.5 GHz) and the UN-II band (5 GHz) [27]. Nowadays, 
WLAN devices are deployed in a wide variety of environments. The importance of harvesting energy from such widely used devices is indisputable. The aim of this study is to design and analysis of the proposed metamaterial structure in order to reveal the potentials as an energy harvester. For this purpose, a textile based metamaterial perfect absorber is designed and simulated to calculate the absorption value at $5 \mathrm{GHz}$ operating frequency.

\section{Design and Simulation}

The unit cell and the periodic array of the proposed MTM structure is given in Figure 1. The structure is composed of PCPTF substrate, felt dielectric layer, and PCPTF resonator. The thickness values of both the PCPTF substrate and resonator are 0.035 $\mathrm{mm}$. The electrical conductivity, measured resistance, and surface resistance of PCPTF are $2.510^{5} \mathrm{~S} / \mathrm{m}, 0.031 \Omega / \mathrm{sq}$, and $0.05 \Omega / \mathrm{sq}$, respectively. On the other hand, the dielectric layer, felt has an $\varepsilon$ value of $1.44, \mu$ value of 1 , and tangent delta of 0.044 . The parameters of the structure are given in Table 1.

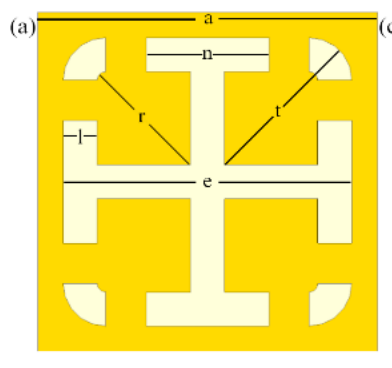

(b)

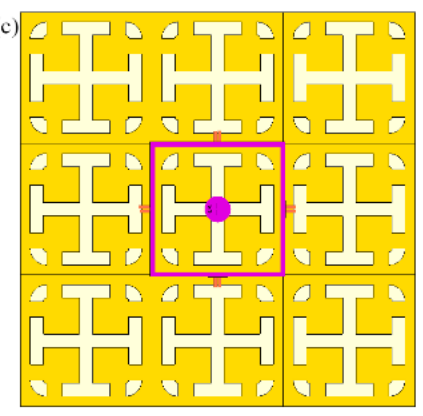

Fig. 1 (a) The front view, (b) the top view, and (c) the periodic array of the proposed structure

Table 1 The parameters of the structure

\begin{tabular}{cc}
\hline Parameter & Value $(\mathrm{mm})$ \\
\hline $\mathrm{a}$ & 20.00 \\
$\mathrm{n}$ & 7.24 \\
$\mathrm{r}$ & 7.50 \\
$\mathrm{t}$ & 9.50 \\
$\mathrm{l}$ & 2.00 \\
$\mathrm{e}$ & 17.00 \\
\hline
\end{tabular}

The simulation was conducted by a full-wave EM solver CST Microwave Studio (Computer Simulation Technology $\mathrm{GmbH}$, Darmstadt, Germany) based on finite integration technique. 3.5-6.5 GHz frequency range were chosen for calculations. The unit cell was chosen as the boundary conditions for $X_{\min }, X_{\max }$ and $Y_{\min }, Y_{\max }$. Besides, the Floquet boundaries were preferred by choosing $\mathrm{Z}_{\min }$ and $\mathrm{Z}_{\max }$ as open (add space) areas. The absorption equation used is

$$
A(\omega)=1-R(\omega)-T(\omega)
$$

Since $R(\omega)=\left|S_{11}\right|^{2}$ and $\mathrm{T}(\omega)=\left|S_{21}\right|^{2}$, the $\left|S_{11}\right|$ and $\left|S_{21}\right|$ values should be minimized to raise the absorption value. Therefore, the substrate material was chosen as PCPTF and transmission value was minimized by this means. The reflection of the MTM structure 
is tried to be minimized by adjustment of $S_{11}$ parameter. The absorption of the proposed MTM structure was calculated and presented as graph. In addition, the permittivity, the permeability, and the refractive index were given.

\section{Results and discussion}

\subsection{Permittivity, permeability, and refractive index of the proposed structure}

In an effective medium, MTMs are described by complex electric permittivity $\left(\tilde{\epsilon}(\omega)=\epsilon_{1}+i \epsilon_{2}\right)$ and magnetic permeability $\left(\tilde{\mu}(\omega)=\mu_{1}+i \mu_{2}\right)$. Since the EM properties of MTMs with a negative refractive index can be easily controlled by the components constituting the MTM structure and by the geometric parameters, these parameters can be adjusted according to the applications. The frequency dependent transmission, $T(\omega)$ and reflection, $R(\omega)$ of a MTM are the parameters affecting the absorption of the MTM. As stated in Eq. (1), maximizing the absorption, $A(\omega)$ is possible via minimizing $T(\omega)$ and $R(\omega)$, simultaneously at relevant frequency.

Fig. 2 shows the effective parameters and refractive index of proposed MTM structure at between 3.5 and $6.5 \mathrm{GHz}$. Figure 2 denotes that the values of the real $\varepsilon, \mu$ and $n$ are $0.25,-0.28$, and -0.15 , respectively at the resonance frequency. The most important MTM property is a negative refractive index for real part, and this proves that the proposed structure provides this property at the $5 \mathrm{GHz}$ frequency range.

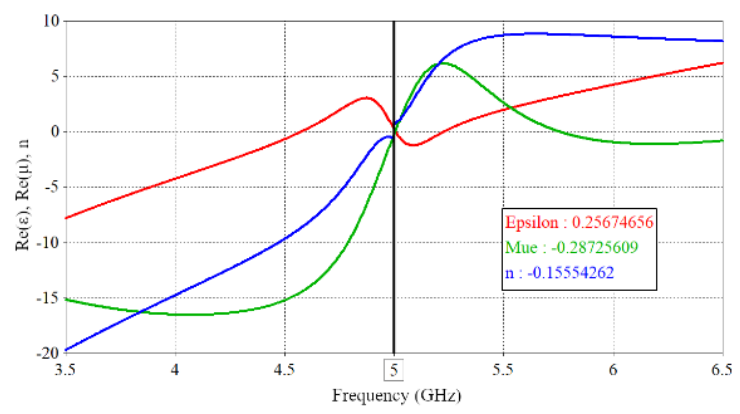

Fig. 2 Permittivity $(\varepsilon)$, permeability $(\mu)$, and refractive index (n) of the proposed MTM structure

\subsection{The absorption, reflection, and transmission of the proposed structure}

The absorption, reflection, and transmission graph of the proposed MTM structure at $3.5-6.5 \mathrm{GHz}$ is given in Figure 3. There was only one peak observed at the frequency range considered. The proposed MTM structure has an absorption value of $99.49 \%$ as seen. This value indicates that the designed structure is a perfect absorber and has potentials to be used in energy harvesting applications. 


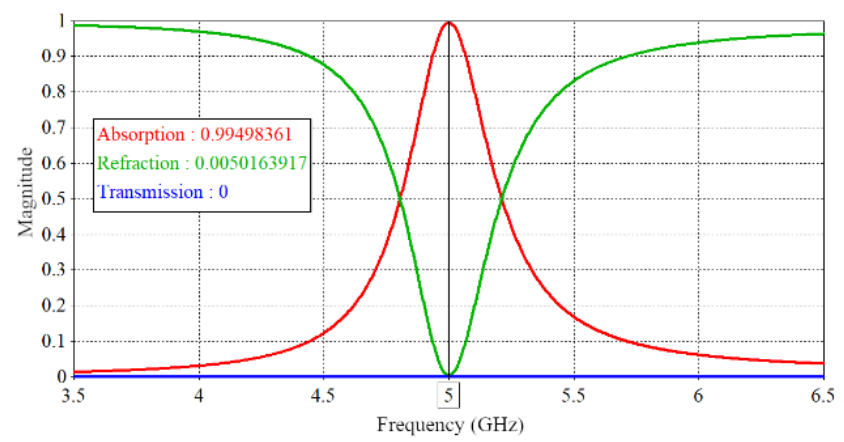

Fig. 3 Variation of absorption, transmission, reflection of the proposed MTM structure.

\subsection{Harvesting application}

The basis of energy harvesting studies is that the MTM provides excellent absorption of incoming waves in the desired frequency range. In addition, the MTM-based energy harvesting systems also require the highest power distribution to a load to ensure that the absorbed power is spread across the load. According to energy harvesting researches, energy harvesting devices can harvest several types of energy and convert EM energy into electrical energy.

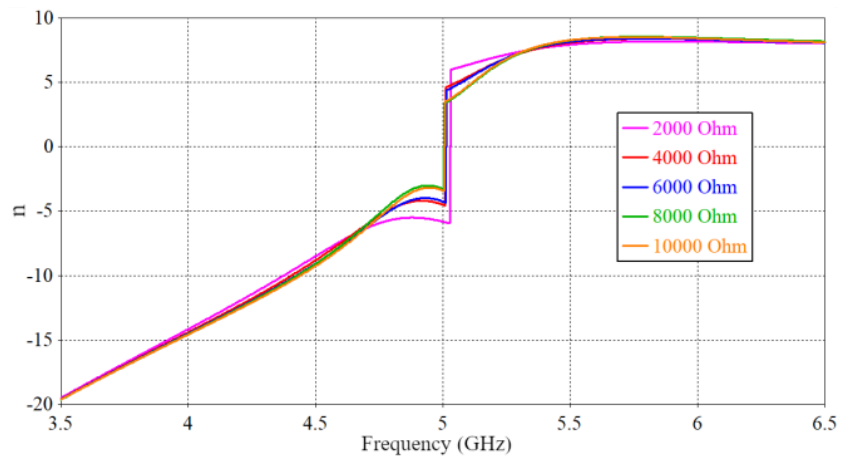

Fig. 4 Refractive index (n) of the proposed MTM structure in the 2000-10000 $\Omega$ range (in $2000 \Omega$ increments)

The refractive index characteristics of the proposed MTM structure have been examined considering the resistor values in 2000-10000 $\Omega$ range in $2000 \Omega$ increments. The proposed structure has refractive index values of - 5.50, -4.22, -4.03, 3.08 , and -3.24 at $2000,4000,6000,8000$, and $10000 \Omega$ for each resistor value, respectively (Fig. 4). The negative refractive index values obtained denote that the structure with added resistance also exhibits MTM characteristics. Moreover, the frequency values for these resistor values were obtained as 4.88, 4.94, 4.96, 4.98, and $4.97 \mathrm{GHz}$, which were very close to the resonant frequency. This discloses that the proposed MTM has a good yield in harvesting applications. 


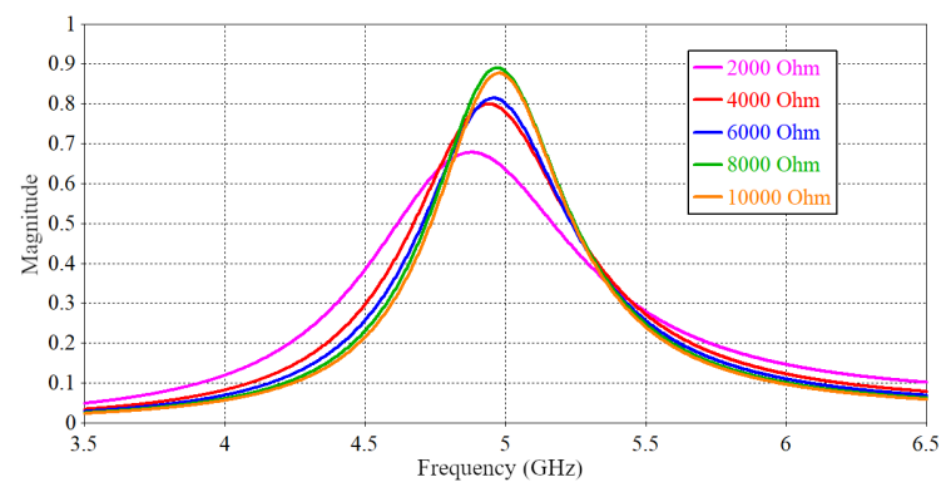

Fig. 5 The absorption of the proposed MTM structure in 2000 and $10000 \Omega$ range (in $2000 \Omega$ increments)

In Fig. 5, the absorption characteristics of the MTM structure have been investigated according to the abovementioned resistor values. The structure has absorption percentages of $67.8,80.3,81.1,88.3$, and $87.6 \%$ at the frequencies $4.81,4.92,4.96$, 4.98, and $4.97 \mathrm{GHz}$ for each resistor value, respectively. Fig. 5 shows that the absorption is maximum in $8000 \Omega$ resistor value. The overall results show that this structure exhibits perfect absorption properties and have potentials to be used in the perfect absorption based harvesting applications.

\section{Conclusion}

It was aimed to perform a design and simulation study of a MTM perfect absorber and to present the harvesting efficiency of this structure by simulation analysis. For this purpose, a textile based MTM unit cell was designed with three layers as PCPTF substrate, felt dielectric layer, and the PCPTF resonator. The substrate layer was chosen as PCPTF to minimize the transmission value. The reflection value was minimized by accurate selection of material type and the geometrical design. According to the simulation analysis results, the refractive index was negative, and the absorption value was $99.49 \%$ at $5 \mathrm{GHz}$ frequency band. The refractive index of the proposed MTM structure was investigated according to the resistor values in 2000-10000 $\Omega$, in $2000 \Omega$ increments. And the frequency values obtained in this range were very close to $5 \mathrm{GHz}$ showing a good yield. When the absorption characteristics were taken into consideration, it was seen that the maximum absorption was obtained as $88.3 \%$ in $8000 \Omega$ resistor value. The overall results of this study reveal that the proposed MTM structure is a good candidate for energy harvesting applications.

\section{Acknowledgement}

The authors thank to Erkan TETIK (PhD) for his support in CST analyses.

\section{References}

1. Chilabi HJ, Salleh H, Al-Ashtari W, Supeni EE, Abdullah LC, As'arry AB, Rezali KAM, Azwan MK. Rotational piezoelectric energy harvesting: a comprehensive review on excitation elements, designs, and performances. Energies. 2021;14(11):3098. 
2. Karakaya E, Bagci F, Yilmaz AE, Akaoglu B. Metamaterial-based four-band electromagnetic energy harvesting at commonly used GSM and Wi-Fi frequencies. J. Electron. Mater. 2019;48:2307-2316.

3. Unal E, Dincer F, Tetik E, Karaaslan M, Bakir M, Sabah C. Tunable perfect metamaterial absorber design using the golden ratio and energy harvesting and sensor applications. J. Mater. Sci: Mater. Electron.2015;26:9735-9740.

4. Veselago VG. The Electrodynamics of substances with simultaneously negative values of $\varepsilon$ and $\mu$. Sov. Phys. Uspekhi. 1968.

5. Al-badri KSL. Ultrathin perfect metamaterial absorber based on triquetra shape. IOP Conf. Ser.: Mater. Sci. Eng. 2020;928,072065.

6. Bağmancı M, Karaaslan M, Ünal E, Akgol O, Sabah C. Extremely-broad band metamaterial absorber for solar energy harvesting based on star shaped resonator. Opt. Quantum Electron. 2017;49:1-14.

7. Lee W, Choi SI, Kim HI, Hwang S, Jeon S, Yoon YK. Metamaterial-integrated high-gain rectenna for RF sensing and energy harvesting applications. Sensors (Basel). 2021;21(19):6580.

8. Bakir M, Karaaslan M, Altintas O, Bağmancı M, Akdogan V, Temurtas F. Tunable energy harvesting on UHF bands especially for GSM frequencies. Int. J. of Microw. Wirel. Technol. 2018;10(1):67-76.

9. Elwi TA, Jassim DA, Mohammed HH. Novel miniaturized folded UWB microstrip antenna-based metamaterial for RF energy harvesting. Int. J. Commun. Syst. 2020;33:e4305.

10. Pandey R, Shnakhwar AK, Singh A. Design, analysis, and optimization of dual side printed multiband antenna for energy harvesting applications. Prog. Electromagn. Res. C. 2020;102:79-91.

11. Dincer F. Electromagnetic energy harvesting application based on tunable perfect metamaterial absorber. J. Electromagn. Waves Appl. 2015;29,18:24442453.

12. Das R, McGlynn E, Yuan M, Heidari H. Serpentine-shaped metamaterial energy harvester for wearable and implantable medical systems. 2021 IEEE Int. Symp. Circuits Syst. 2021;1-5.

13. Mulazimoglu C, Karakaya E, Can S, Yilmaz AE, Akaoglu B. Hexagonal-shaped metamaterial energy harvester design. 10th Int. Congr. Adv. Electromagn. Mater. Microw. Opt. 2016; 82-84.

14. Hossain MJ, Faruque MRI, Islam MT, Mat KB. A new compact octagonal shape perfect metamaterial absorber for microwave applications. Appl. Sci. 2017;7(12):1263.

15. Tetik E. Flexible perfect metamaterial absorber and sensor application at terahertz frequencies, J. Optoelectron. Adv. Mater. 2020;22(5-6):213-218.

16. Tetik E, Antepli A. The effect on the human body of a wearable circular antenna based on metamaterial. 5th Int. Conf. Mater. Sci. Adv. Nanotechnologies Nxt Gen. 2018.

17. Koski K, Vena A, Sydänheimo L, Ukkonen L, Rahmat-Samii Y. Design and implementation of electro-textile ground planes for wearable UHF RFID patch tag antennas. IEEE Antennas Wirel. Propag. Lett. 2013;12:964-967. 
18. Yang S, Thacker Z, Allison E, Pinhero P. Optimization of three-dimensional metamaterials for terahertz energy harvesting. J Vac. Sci. Technol. A 2021;39, 062801.

19. Sankar VS, Kumari R, Choudhury B. Metamaterial-inspired rectenna loaded with a DR for RF energy harvesting. 2018 IEEE Indian Conf. Antennas Propag. (InCAP). 2018;1-3.

20. Alkurt FO, Altintas O, Bakir M, Tamer A, Karadag F, Karaaslan M, Unal M, Akgol O. Octagonal shaped metamaterial absorber based energy harvester. Mater. Sci. 2018;24(3).

21. Akarsu G, Nakmouche MF, Fawzy DE, Allam AMMA, Başköy K, Cengiz MF. A novel $5 \mathrm{G}$ wideband metamaterial based absorber for microwave energy harvesting applications. 8th International Conference on Electrical and Electronics Engineering (ICEEE). 2021;309-312.

22. Kaur KP, Upadhyaya T, Palandoken M, Gocen C. Ultrathin dual-layer tripleband flexible microwave metamaterial absorber for energy harvesting applications. Int. J. RF Microw. Comput. Aided Eng. 2019;29:e21646.

23. Coskuner E, Garcia-Garcia JJ. Metamaterial impedance matching network for ambient RF-energy harvesting operating at $2.4 \mathrm{GHz}$ and $5 \mathrm{GHz}$. Electronics. 2021;10(10):1196.

24. Aldhaeebi MA, Almoneef TS. Planar dual polarized metasurface array for microwave energy harvesting. Electronics. 2020;9(12):1985.

25. Costanzo S, Venneri F. Polarization-insensitive fractal metamaterial surface for energy harvesting in IoT applications. Electronics. 2020;9(6):959.

26. Umaña-Idarraga F, Cataño-Ochoa D, Montoya-Villada S, Valencia-Balvin C, Reyes-Vera E. Design of a perfect and multi-resonant metamaterial absorber for electromagnetic energy harvesting applications. J. Phys.: Conf. Ser. $2021 ; 2118$.

27. Ruppel CCW, Reindl L, Weigel R. SAW devices and their wireless communications applications. IEEE Microw. Mag. 2002;3(2):65-71. 